\title{
Le cas Stassen : un maître illustrateur au cœur du premier cercle wagnérien
}

Jean-Louis Elloy

\section{OpenEdition}

1 Journals

Édition électronique

URL : https://journals.openedition.org/rbnu/2373

DOI : $10.4000 /$ rbnu. 2373

ISSN : 2679-6104

Éditeur

Bibliothèque nationale et universitaire de Strasbourg

\section{Édition imprimée}

Date de publication : 1 mai 2013

Pagination : 86-101

ISBN : 9782859230432

ISSN : 2109-2761

\section{Référence électronique}

Jean-Louis Elloy, «Le cas Stassen : un maître illustrateur au cœur du premier cercle wagnérien », La Revue de la BNU [En ligne], 7 | 2013, mis en ligne le 01 mai 2013, consulté le 08 août 2021. URL : http:// journals.openedition.org/rbnu/2373; DOI : https://doi.org/10.4000/rbnu.2373

\section{(c) (i) (8)}

La Revue de la BNU est mise à disposition selon les termes de la Licence Creative Commons Attribution - Pas d'Utilisation Commerciale - Partage dans les Mêmes Conditions 4.0 International. 


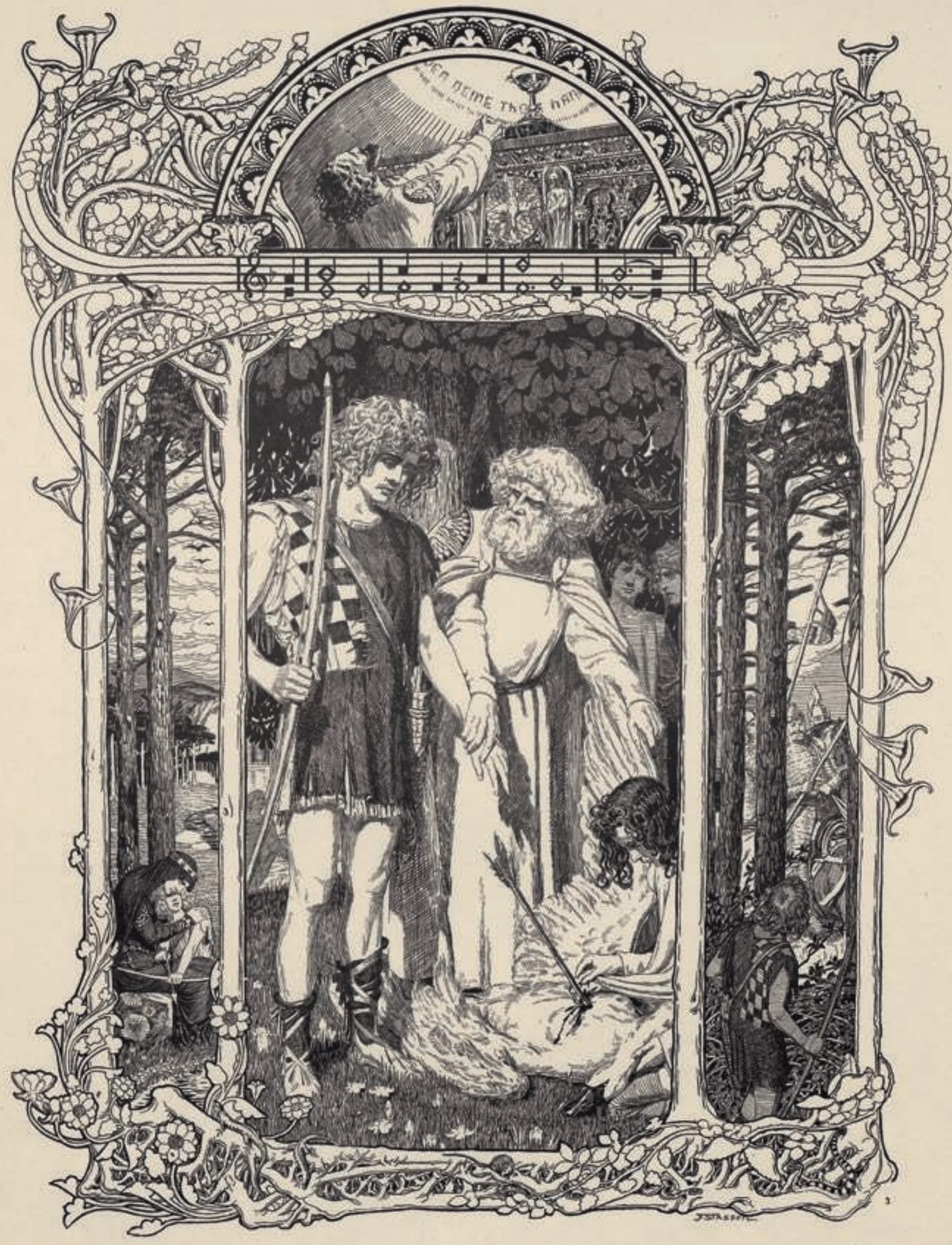




\section{Le cas Stassen :}

\section{un maître illustrateur}

au cœur du premier cercle wagnérien

Le riche fonds Boulet-Devraigne récemment acquis par la BNU témoigne du foisonnement iconographique qu'a suscité l'œuvre de Wagner dans les décennies qui ont suivi sa mort. Les illustrations de ce fonds sont de nature esthétique très diverse tant par leur caractère que par leur projet. Certaines présentent un lien étroit avec les premières mises en scène de Bayreuth, comme les lithographies en couleurs reproduisant les costumes dessinés par Carl Emil Doepler pour la création du Ring à Bayreuth en 1876. D'autres tendent à vulgariser l'univers dramatique des œuvres de Wagner à travers une imagerie faussement médiévale plus ou moins inspirée des décors retenus pour ces mêmes circonstances. Les gravures de Ferdinand Leeke, exemple emblématique, ont grandement contribué, par leur large diffusion, à développer cette veine de tableaux historicisants, involontairement caricaturale et très vite elle-même objet de caricature dans la presse satirique fustigeant le wagnérisme. Aujourd'hui encore, sans doute pour son charme kitsch, Leeke est l'artiste le plus reproduit sur les cartes postales vendues dans les stands du Festspielhaus. Mais le fonds Boulet-Devraigne abonde en œuvres plus ambitieuses d'artistes qui, souvent fascinés par les drames de Wagner, y ont trouvé une source d'inspiration personnelle : c'est le cas des gravures de Fantin-Latour, des livres illustrés d'Arthur Rackham, des planches de Hermann Hendrich, de celles de Hugo Braune et surtout des suites gravées présentées en portefeuilles de Franz Stassen, objets artistiques les plus spectaculaires de ce fonds.
Ces dernières méritent une attention particulière à plus d'un titre. Le nom de Stassen et son univers sont familiers à tout wagnérien. Ses Siegfried, ses Parsifal, ses Brünnhilde et autres filles du Rhin agrémentent bien souvent, en vignettes décoratives, les divers programmes d'opéra ou les publications illustrées sur Wagner et son œuvre. Les gravures d'origine se sont ainsi disséminées dans un kaléidoscope bayreuthien suranné, un livre d'images devenu familier qui finit par se confondre avec les scènes héroïques à la Leeke. Mais cette dispersion profuse de reproductions - surprenant paradoxe pour un artiste qui a construit sa carrière sur la production commerciale de l'image - ne permet ni d'apprécier la cohérence de son projet wagnérien car on peut le considérer comme tel - ni d'en mesurer la qualité esthétique. Il faut pour cela avoir le loisir d'embrasser du regard les planches dans leur format premier, dans la continuité et l'intégralité des scènes et de leurs cycles. Seule une exposition peut offrir cette occasion. A plusieurs reprises le Richard-WagnerMuseum de Bayreuth a montré au public une part du large fonds Stassen dont il dispose. La ville natale de Stassen, Hanau, a également organisé en 1999 une rétrospective, et à Paris on a pu voir ses illustrations du Ring en $1994^{1}$. C'est peu pour le faire réellement connaître, d'autant que les commentaires - en France, surtout - restent souvent très laconiques et prudents dans leurs jugements ou leurs analyses ${ }^{2}$. De fait, la dérive idéologique de Stassen, qui d'un pangermanisme convaincu l'a conduit à adhérer au nazisme et à même 
devenir artiste officiel du régime (non sans un certain nombre de malentendus), a entaché la réception de son œuvre ${ }^{3}$. Cette dérive est également esthétique : le conservatisme toujours plus prégnant de son expression artistique ne peut que détourner le public d'aujourd'hui de l'essentiel de ses lithographies et plus encore de ses tableaux. Mais sa production, dans les années 1900, des premières gravures inspirées de Wagner, se prête à un autre regard. Elle est riche d'ambitions qu'on ne retrouvera plus chez Stassen, ni ensuite ni ailleurs pendant la même période.

On retiendra ici principalement trois centres d'intérêt qui peuvent justifier un examen plus atten-

tif de ces gravures : la tentative d'illustrer le drame musical de Wagner et non le seul fil dramatique de ses livrets, la mise à contribution, pour ce faire, de l'esthétique Jugendstil, et le halo idéologique de ce projet, révélateur de la relecture de Wagner orchestrée par le premier cercle bayreuthien.

Richard Wagner tient une place essentielle dans la vie de Stassen. Si ce peintre et dessinateur n'a pas connu personnellement le musicien, il lui a consacré très tôt des illustrations remarquées et n'a cessé ensuite jusque dans ses dernières années de s'inspirer de Wagner dans ses tableaux, ses gravures, ses lithographies. A partir de 1908, il fréquente assidûment le cercle de Bayreuth, en devient un membre très actif, se lie intimement à Siegfried Wagner (il participera à l'édition de sa biographie publiée en 1911), à Hans von Wolzogen ${ }^{4}$ et encore à d'autres sectateurs du culte wagnérien, sans oublier l'inévitable Cosima. Le fonds Boulet-Devraigne possède ses ensembles graphiques les plus importants, ceux que l'artiste lui-même considérait comme ses œuvres les plus personnelles ${ }^{5}$ : le cahier grand format des planches consacrées à Tristan et Isolde, paru en 1899, sa suite Parsifal de 1901/1902, ses lithographies illustrant le Ring, travail qui s'échelonne de 1908 à $1930^{6}$. Ces portfolios ont été publiés chez Fischer et Franke pour les premiers, puis chez l'éditeur Ludwig Schroeter (à partir de La Walkyrie), ami d'enfance et conseiller artistique de Stassen.
Tristan et Isolde a, dès sa parution, remporté un large succès et promu son auteur au rang des illustrateurs Jugendstil les plus appréciés de son époque. Pourtant le ralliement de Stassen à cette esthétique nouvelle a été loin d'être évident et immédiat. Formé au départ dans l'art du dessin à la Kunstschule de Hanau (dans la région de Francfort), sa ville natale, il reçoit une éducation artistique très académique, tournée vers les scènes historiques et bibliques, l'imitation des modèles antiques ou de la Renaissance italienne. Fortement influencé par le directeur de cette école, F. K. Hausmann, et par G. Cornicelius auprès duquel il suit des cours privés, il bénéficie d'un enseignement tenu alors pour l'un des meilleurs d'Allemagne, mais peu ouvert aux novateurs. Son parcours le conduit ensuite à la Berliner Hochschule für bildende Künste dont la figure dominante, celle du peintre d'histoire Anton von Werner, va le conforter dans une voie très classique.

Mais dans le même temps Stassen s'éveille au Jugendstil. Il séjourne brièvement à Munich après s'être acquitté de ses obligations militaires en 1892. S'il reste étranger à l'effervescence des revues et de la société artistiques de la ville, il n'en apprend pas moins à les connaître. Lorsqu'il revient quelques mois plus tard à Berlin où il loue un atelier, il rencontre Fidus (Hugo Höppener), Melchior Lechter (illustrateur d'élection de Stefan George) et multiplie les illustrations de livres qui font très tôt sa célébrité. Il s'y réfère au Ver Sacrum, la fameuse revue Jugendstil viennoise, ou encore à William Morris et John Ruskin, par des choix décoratifs géométriques et floraux caractéristiques. C'est aussi sous l'influence de Sascha Schneider qu'il décide de renoncer aux petits formats pour adopter celui de grands cartons à dessins. De tous ces croisements humains et artistiques, nous avons le témoignage à la fois par le tournant stylistique pris par Stassen à la fin des années 1890 et par son récit et ses commentaires dans le texte autobiographique qu'il publie en 1938. Dans ce texte (Mein Leben), il nous décrit aussi son enthousiasme pour les peintres nazaréens (en particulier Steinle, Veit et Overbeck) qu'il découvre au Städelsches Institut de 
Francfort. Eux non plus ne sont pas sans effet sur l'iconographie wagnérienne de Stassen.

Pendant quelques années, il participe aux expositions de la Sécession berlinoise avec des toiles aux titres éloquents : Flora, Pan rêve, pour n'en citer que les plus explicitement portés par l'esprit nouveau. Il semble toutefois que le refus d'une de ses œuvres (Iphigénie à Aulis) l'ait détourné de la Sécession. De fait, à la suite de cet échec, il devient membre du Verein Berliner Künstler et se replie à partir des années 1900 dans un conservatisme de plus en plus marqué d'idéologie nationaliste et pangermanique. Les œuvres inspirées de Wagner garderont pourtant assez longtemps l'empreinte d'un détour par le Jugendstil.

Revenons-en aux ambitions musicales et wagnériennes de Stassen. Suivant la voie du "Gesamtkunstwerk " (l'œuvre d'art totale), notion centrale des principaux essais de Wagner sur le drame musical, Stassen a cherché à allier différentes formes d'expression artistique, en l'occurrence la musique et l'image.

Prenons pour exemple son Parsifal. L'œuvre de Wagner, qui comporte par elle-même peu d'événements dramatiques, se prête mal au déploiement d'une imagerie épique. Le cycle graphique n'a donc que très peu à raconter. Il retient bien sûr quelques scènes clés qui dans l'ensemble conservent le déroulement linéaire du livret. Mais le travail artistique porte autant sur la mise en page de la planche tout entière que sur l'encadré central figurant la scène.

Chacune des quinze gravures du cycle retient un moment emblématique de l'opéra (la mort du cygne, l'arrivée d'Amfortas, Klingsor missionnant Kundry...). Chacune s'affiche dans un portique stylisé avec un degré d'abstraction variable selon les scènes. Les pourtours latéraux souvent riches en linéatures très dynamiques ainsi que, selon les cas, le cartouche inférieur ou supérieur, tout comme parfois les tondi diversement situés, font surgir des scènes annexes en corrélation temporelle (par prolepse ou analepse) ou symbolique avec le dispositif central. Le regard du spectateur peut ainsi suivre un double cheminement : un parcours horizontal lui retrace la structure dramatique de l'œuvre de Wagner, et dans le même temps un élan vertical figure la rédemption mystique qu'elle signifie. Que la mystique de Stassen ne soit pas tout à fait celle de Wagner, peu importe. Ce que l'illustrateur emprunte au musicien, c'est la coïncidence entre une approche narrative et une visée interprétative. La scène, simultanément récit et exégèse, raconte et révèle à la fois. La troisième gravure par exemple représente en son centre Parsifal venant de tuer le cygne. La lecture horizontale rappelle les événements passés de l'enfance du héros : il quitte sa mère Herzeleide (dans le bandeau latéral gauche du cadre); il rencontre les chevaliers (dans le bandeau latéral droit). La lecture verticale établit des correspondances symboliques entre ces épisodes et au-delà d'eux. Le cartouche supérieur représente Amfortas souffrant et priant, et en hauteur, le Graal et sa prophétie de l'arrivée d'un « reine Tor" (un pur fol). Dans une agogique ascendante, la douleur maternelle est ainsi associée visuellement et significativement à celle du cygne transpercé par la flèche, qui renvoie elle-même à celle d'Amfortas blessé par la lance tandis que le regard compatissant de Parsifal sur le cygne fait de lui, dès ce premier acte de présence, le héros de la rédemption par la compassion, l'élu du Graal, déjà roi des chevaliers.

Il s'ensuit que le présent de la scène porte en lui passé et futur. On voit bien que Stassen cherche dans sa composition graphique une sorte d'équivalent au traitement du temps musical dans le drame wagnérien, un temps aussi bien vertical (qui procède par superposition de strates temporelles grâce au jeu des combinaisons thématiques) qu'horizontal (c'est-à-dire simplement linéaire).

Dans l'édition de 1914, cet intérêt porté aux ébrasements du temps parsifalien est affiché d'entrée. Un poème liminaire de Wolzogen y rend hommage à Wagner. Wolzogen y reformule ce vers de l'acte I de Parsifal, si souvent commenté depuis, "Zum Raum wird hier die Zeit " (ici le temps se fait espace) et l'annonce ainsi comme éclairant également la composition de Stassen. L'illustrateur, en effet, le fait sien à plusieurs reprises. La planche 4, par exemple, figure le moment où Gurnemanz prononce ce vers alors qu'il conduit Parsifal à Montsalvat. Dans l'opéra, l'espace semble miraculeusement se réduire de lui-même et bouleverser la perception du temps ; sur la gravure, le procédé est inverse mais vise à produire le même effet. Le surgissement d'une profonde perspective, unique dans le cycle, met tout à coup à distance le temple du Graal, si proche dans les médaillons des gravures précédentes. Le plan large donne à voir la durée du cheminement initiatique. Dans la première planche, par contre, c'est l'abolition de la durée que traduit l'espace intemporel de la nuit. On y voit Titurel en prière recevant des anges la coupe sacrée, dans une attitude et avec des traits préfigurant Amfortas ou Parsifal découvrant le Graal, et annonçant ainsi la 


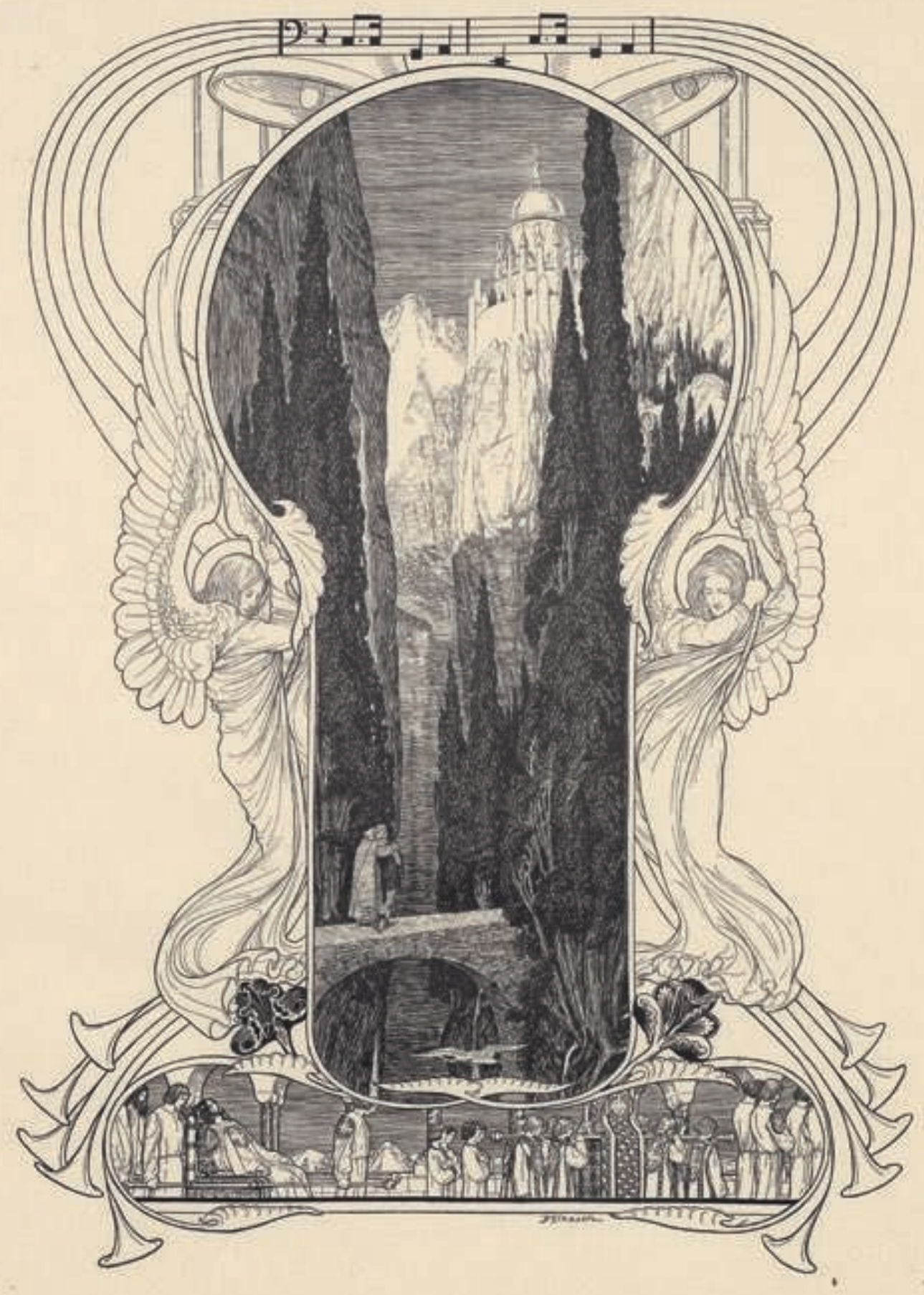




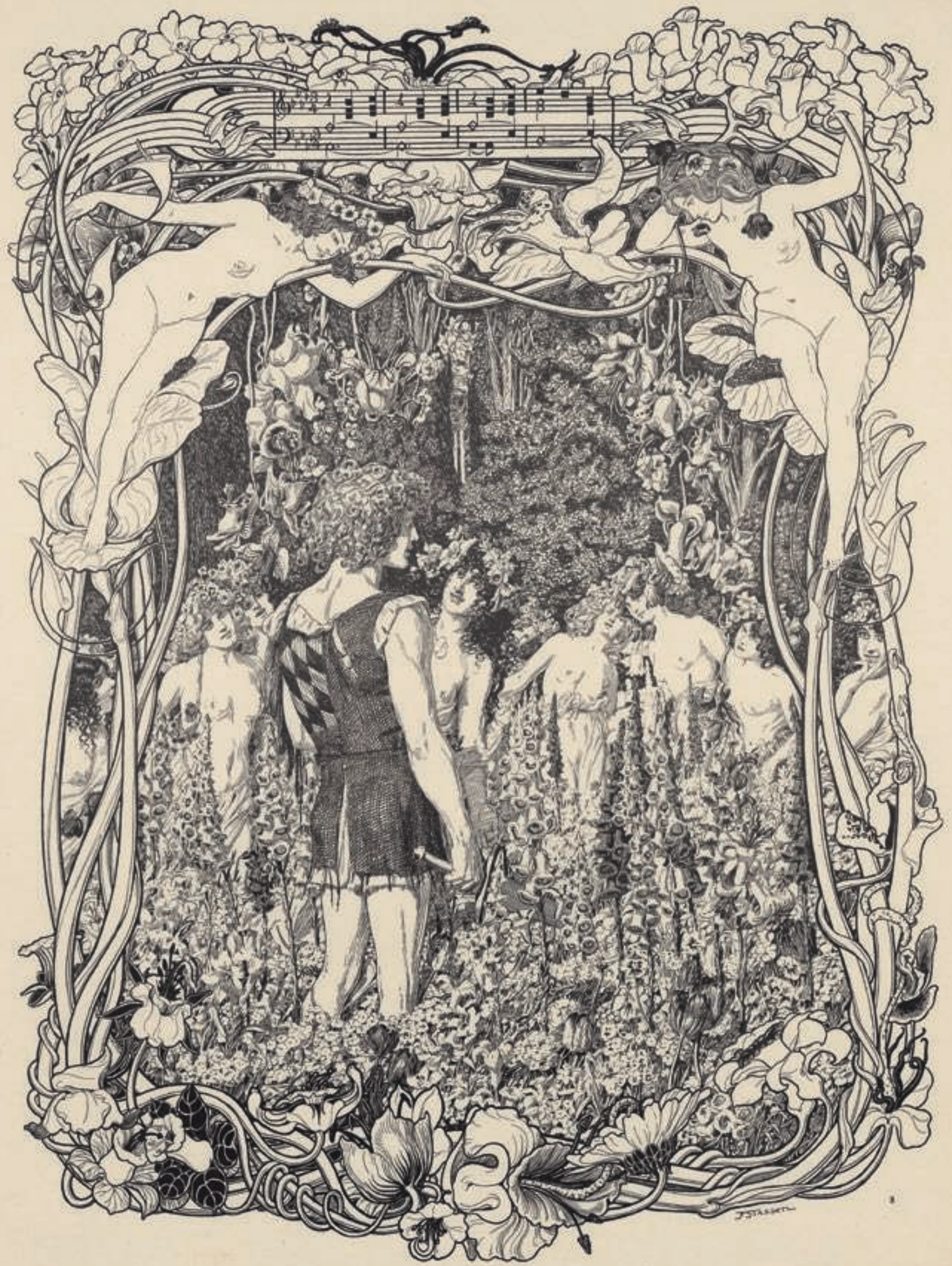


perpétuation atemporelle de la communion mystique.

C'est Wolzogen, on le sait, qui a établi les premiers répertoires des leitmotive wagnériens et qui les a dénommés. Dès 1882, il en propose un catalogue pour Parsifal. Il n'est donc pas étonnant que le cycle de Stassen leur accorde une place majeure et, là encore, grâce à un art de la correspondance entre musique et dessin ${ }^{7}$. Les leitmotive chez Stassen reprennent quelques thèmes fondamentaux de la partition dont la notation musicale apparaît à la base ou dans le couronnement de la gravure. Ils s'enrichissent d'une citation du livret explicitant le thème musical ou le dramatisant : cette part textuelle intervient en surimpression sur un feuillet transparent joint à chaque planche. Et dans les gravures elles-mêmes les thèmes sont ensuite déclinés, appariés, transformés. Mais Stassen dépasse la seule intention de mettre en images ou pire encore de cataloguer, comme Wolzogen souhaitait le faire, une panoplie de thèmes-formules qui étiquetteraient le discours musical. Stassen n'est pas loin de retrouver le principe d'une mélodie infinie, ou plus précisément d'une variation continue du motif, caractéristique de l'écriture de Wagner ${ }^{8}$.

Parmi les leitmotive graphiques, on peut mentionner par exemple ceux de la colombe, du Graal, des anges, de la main, du temple, des arbres, de la croix, de la lance, du serpent, des cloches... Ils se répondent les uns aux autres par analogies visuelles dans une perspective d'élévation mystique : de l'arbre à la lance, de la lance à la croix ou de l'ange à la colombe, la transition se déploie avec souplesse ; la figure géométrique du demicercle sert de matrice aussi bien au motif de la coupe du Graal qu'à ceux du portique architectural ou des cloches, selon ses inclinaisons et ses renversements. Le lieu d'apparition du motif varie lui aussi de façon constante : la colombe, par exemple, que Stassen emprunte bien sûr aux didascalies de Wagner, naît dans ses gravures du thème musical de la foi cité dans la planche 1, puis se manifeste dans les lettrines du texte, dans les décors latéraux, les oculi supérieurs, sur le mode de l'ekphrasis comme ornement d'un costume, d'un autel, comme décoration architecturale. Le motif peut encore changer de signification symbolique : celui du temple sacralise Montsalvat ou démonise le royaume de Klingsor ; il nous fait entrer par son portique dans l'espace fictionnel du Bühnenweihfestspiel (festival d'action sacrée), dans la gravure liminaire, puis de plus en plus explicitement dans son espace scénique, les dernières gravures évoquant le décor de Max Brückner pour la représentation de Parsifal à Bayreuth en 1882, et peut-être le bâtiment de Bayreuth lui-même, comme le suggère le poème introductif de Wolzogen.

Les diverses facettes du langage Jugendstil revêtent dans ce cycle elles aussi une fonction musicale. Les fillesfleurs invitaient tout naturellement à une irruption du végétal, de ses lignes courbes et de ses entrelacs sensuels. La planche 8 qui introduit le thème musical des filles-fleurs le visualise par une luxuriance florale représentée dans la scène centrale. A cette expression d'un Jugendstil chargé s'oppose celui de la courbe toujours, mais épurée, abstraite, plus proche cette fois de celle de Peter Behrens que de celle d'August Endell. Cette ligne plus froide dessine par contraste avec les filles-fleurs les figures angéliques, comme venues, elles, de l'univers de Melchior Lechter. Et les encadrements très travaillés de la plupart des planches amplifient encore cette confrontation esthétique. Autrement dit, Stassen intègre à son œuvre l'opposition entre l'ornement et la ligne abstraite, qui divise le Jugendstil au tournant du $20^{e}$ siècle. Cette antithèse structure toute la progression de son cycle : elle traduit la tension entre sensualité et spiritualité ; elle reflète la bipolarité des deux expressions musicales omniprésentes dans le Parsifal de Wagner : celle du chromatisme tout en volutes descendantes des thèmes klingsoriens et celle du diatonisme aux lignes éthérées associé à l'univers du Graal.

Une composition oxymorique comparable préside à l'architecture du cycle consacré à Tristan, cycle de douze planches impressionnantes elles aussi dans leur grand format.

Il paraît en 1899 et précède tout juste celui de Parsifal. Stassen le construit à partir de l'objet, concret et symbolique à la fois, du coffre (" der Schrein "). Dans la fiction du récit, il s'agit du coffret contenant les philtres d'amour et de mort, mais le terme "Schrein " peut aussi désigner le cahier lui-même contenant les gravures. Stassen exploite encore cette polysémie quand, au début des années 1920, il crée son cycle de toiles Weltenwerdenswalterin, Symbol des mütterlichen weiblichen Prinzips (la maîtresse de la destinée des mondes, symbole du principe féminin maternel), dont la deuxième œuvre, Tristanschrein, se présente comme un retable médiéval, autre sens possible du mot "Schrein ». Ce retable d'ailleurs nous fait voir plus nettement encore la démarche esthétique déjà suivie dans son " coffre » de gravures. Les panneaux extérieurs y représentent dans un style historicisant les deux héros 


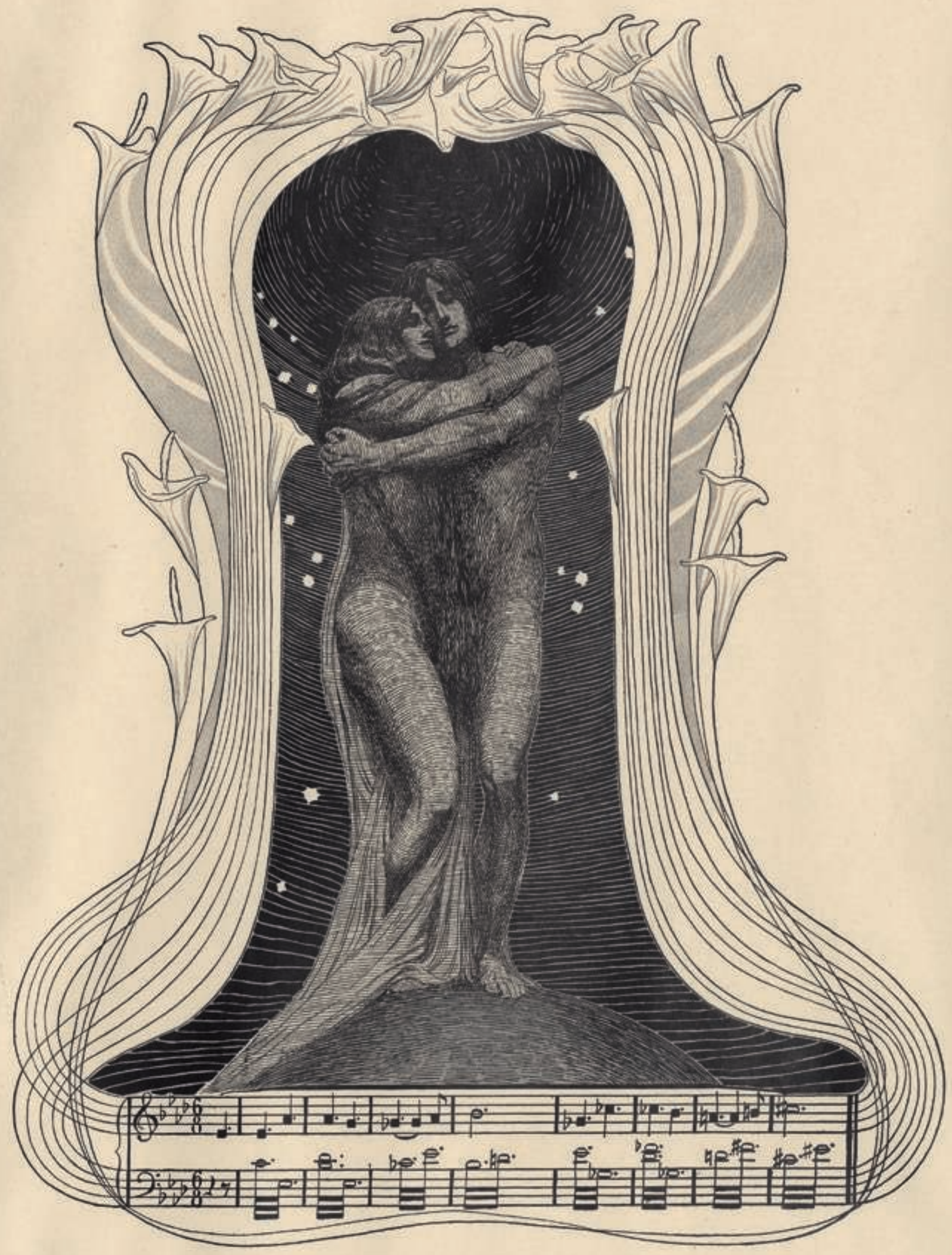


sur le navire qui les conduit chez le roi Marke, Tristan à la barre, Isolde devant sa tente. Au-dessus d'eux, le thème musical de la nostalgie, et au-dessous des zébrures et un crâne annonçant la tragédie. Une fois ouvert, le retable offre au regard trois panneaux où s'affirme l'esthétique tout autre du Jugendstil. Au centre, le couple amoureux s'enlace sous la protection d'un génie de la nuit ; à gauche, Tristan blessé appelle Isolde que l'on voit apparaître sur un tapis floral. Les rinceaux végétaux, la nudité des corps, la stylisation géométrique autant qu'allégorique s'opposent au caractère néo-médiéval et réaliste des peintures extérieures. Ce principe prévaut de manière moins ostensible, mais clairement lisible tout de même, dans le cycle gravé. En son cœur (dans la planche 7) se découvre le royaume de la nuit : les corps nus des amants sont enveloppés d'un pur réseau dynamique de lignes. L'expression Jugendstil, Stassen l'a choisie ici pour dire la force fusionnelle de la passion et l'abstraire du monde social du jour que l'on retrouve, lui, dépeint dans les premières et dernières gravures avec un traditionnel langage historicisant. Dans une logique semblable à celle du cycle de Parsifal, celui de Tristan et Isolde confronte donc deux esthétiques à dessein, non pour agrémenter l'illustration du récit, non parce qu'il s'agirait d'une œuvre de transition hésitant entre deux partis pris artistiques, mais pour signifier graphiquement l'oxymore tragique du jour et de la nuit, clé de l'opéra de Wagner.

On le voit : dans ces deux cahiers gravés, Stassen porte un véritable projet illustrateur. On a évoqué précédemment ses modèles artistiques revendiqués comme tels. Mais en considérant ses suites wagnériennes de 1899 et de 1901, d'autres parentés viennent à l'esprit : on ne peut s'empêcher de penser aux orientations stylistiques et aux ambitions de Heinrich Vogeler et de Max Klinger, deux des plus célèbres dessinateurs et graveurs contemporains de Stassen ${ }^{9}$. Le premier a beaucoup en partage avec Stassen : le goût pour les décors végétaux, les forêts traversées par des chevaliers rédempteurs, les parterres de fleurs, tableaux d'une éternelle reverdie, la renaissance d'un théâtre médiéval idéalisé, le conte immémorial, lavé des impuretés d'un monde social devenu obscur, et fleurant la même fraîcheur naïve que les images russes du premier Kandinsky. Ainsi le Parsifal de Stassen trouve son double dans le Don Quichotte de Vogeler ; les entrelacs Jugendstil de tiges et de linéaments, nés de l'art de l'enluminure, se lovent avec les mêmes arabesques sur les couvertures de la revue Insel et dans les jardins enchantés de Klingsor. Les deux illustrateurs d'ailleurs ont chacun rêvé un temps une utopie artistique, Worpswede ou Bayreuth, sous les lumières d'un créateur tutélaire, Rilke ou Wagner.

Peut-on aller jusqu'à risquer un parallèle entre les gravures de Stassen et celles de Klinger ? Klinger, créateur d'illuminations plus qu'auteur d'illustrations, s'est comme Stassen particulièrement intéressé à la correspondance du visuel et du musical. Par exemple, dans sa suite consacrée à Brahms (la Brahmsphantasie de 1894), vaste cycle de trente-sept feuilles et quarante et une gravures, il associe lui aussi la partition au dessin, varie les effets de mise en page et d'échos. Bien sûr, Klinger s'écarte radicalement de toute retranscription avec un parti pris de liberté audacieux. Ses scènes mi-oniriques mi-réalistes entretiennent avec le texte musical un rapport allégorique plus mystérieux que mystique. En cela, il se distingue de Stassen. Le premier se sert du matériau musical comme portail vers un imaginaire singulier tandis que l'autre bâtit un portail pour servir la singularité d'un imaginaire musical. Pour tous deux, quoi qu'il en soit, la musique joue un rôle déterminant dans la conception graphique.

Après les cycles de Tristan et de Parsifal, Stassen s'attaque au Ring. La publication des quatre cahiers Rheingold (1914), Walküre (1916), Siegfried (1920) et Götterdämmerung (1930) s'étale dans le temps. Ces lithographies en couleurs, mieux connues, ont été très souvent reproduites (mais de façon sélective) et ont fait l'objet de davantage de commentaires. Anton Merk dans son catalogue ${ }^{10}$ souligne l'unité stylistique de l'ensemble, tout en constatant par ailleurs une rupture esthétique flagrante dans l'évolution de l'artiste : le Jugendstil reste présent mais l'ornement, très réduit en comparaison surtout du cycle de Parsifal, n'a plus qu'une maigre fonction décorative ; par contre, le dessin du corps, la plastique de la nudité jouent un rôle plus grand. D’après Anton Merk toujours, ce réalisme corporel rattache encore Stassen au $19^{e}$ siècle. Mais la dramatisation de la gestuelle, les jeux de regards marqués par l'influence de la photographie et du cinéma, tout particulièrement des Nibelungen de Fritz Lang dans les dernières planches (le film est achevé en 1924), le tournent vers la modernité du siècle suivant. On pourrait ajouter qu'il n'est pas impossible non plus qu'auparavant l'illustrateur Stassen ait influencé le cinéaste Lang. Ce dernier ne cite pas les œuvres de Stassen parmi ses sources iconographiques mais la 

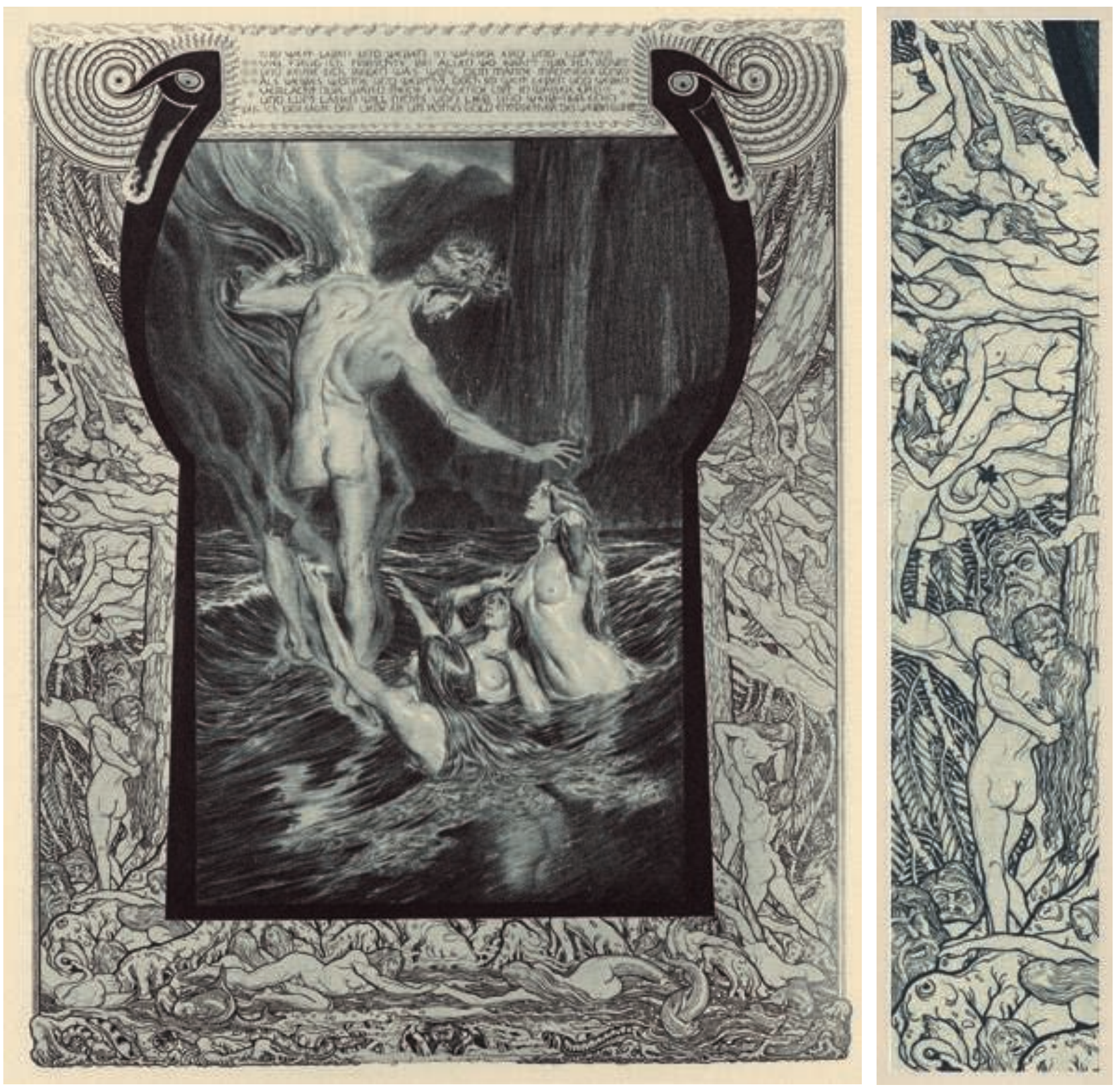

Onzième planche (avec détail) du cycle Der Ring des Nibelungen : Rheingold (Berlin, Ludwig Schroeter, s. d. [ca. 1930] ; coll. BNU) 


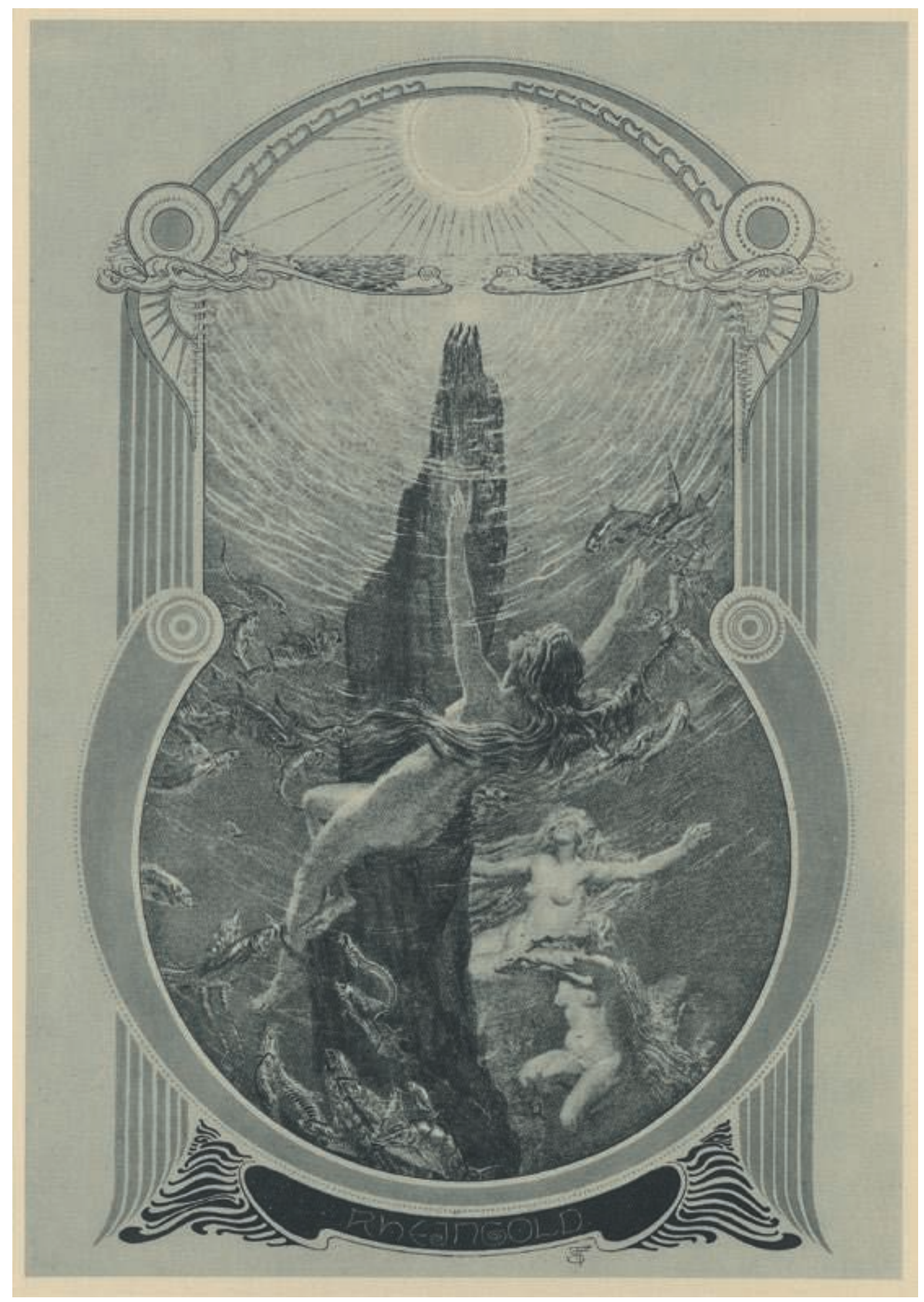

Planches 4, 10, 3 et 2 du cycle Der Ring des Nibelungen: Rheingold

(Berlin, Ludwig Schroeter, s. d. [ca. 1930] ; coll. BNU) 

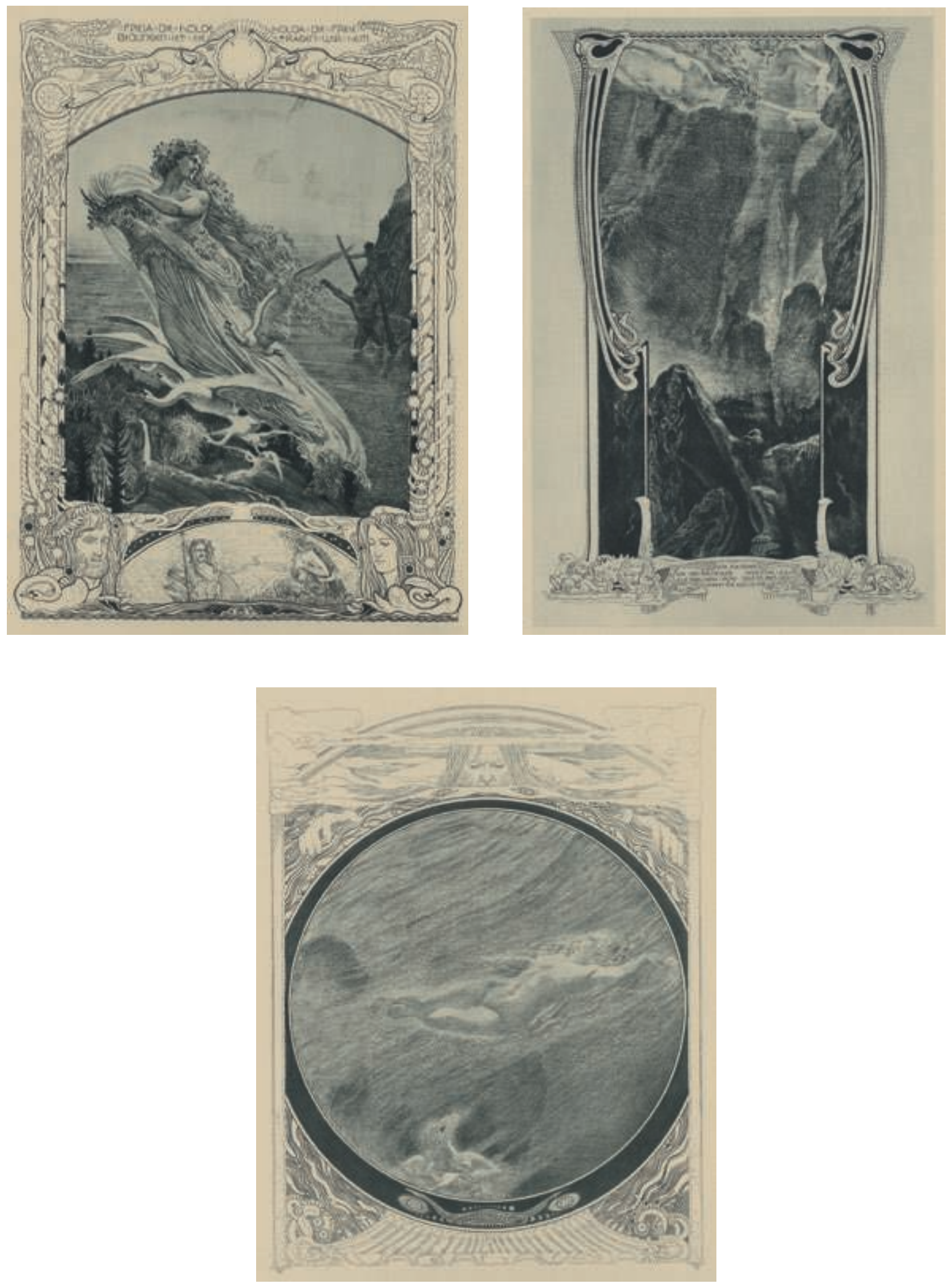


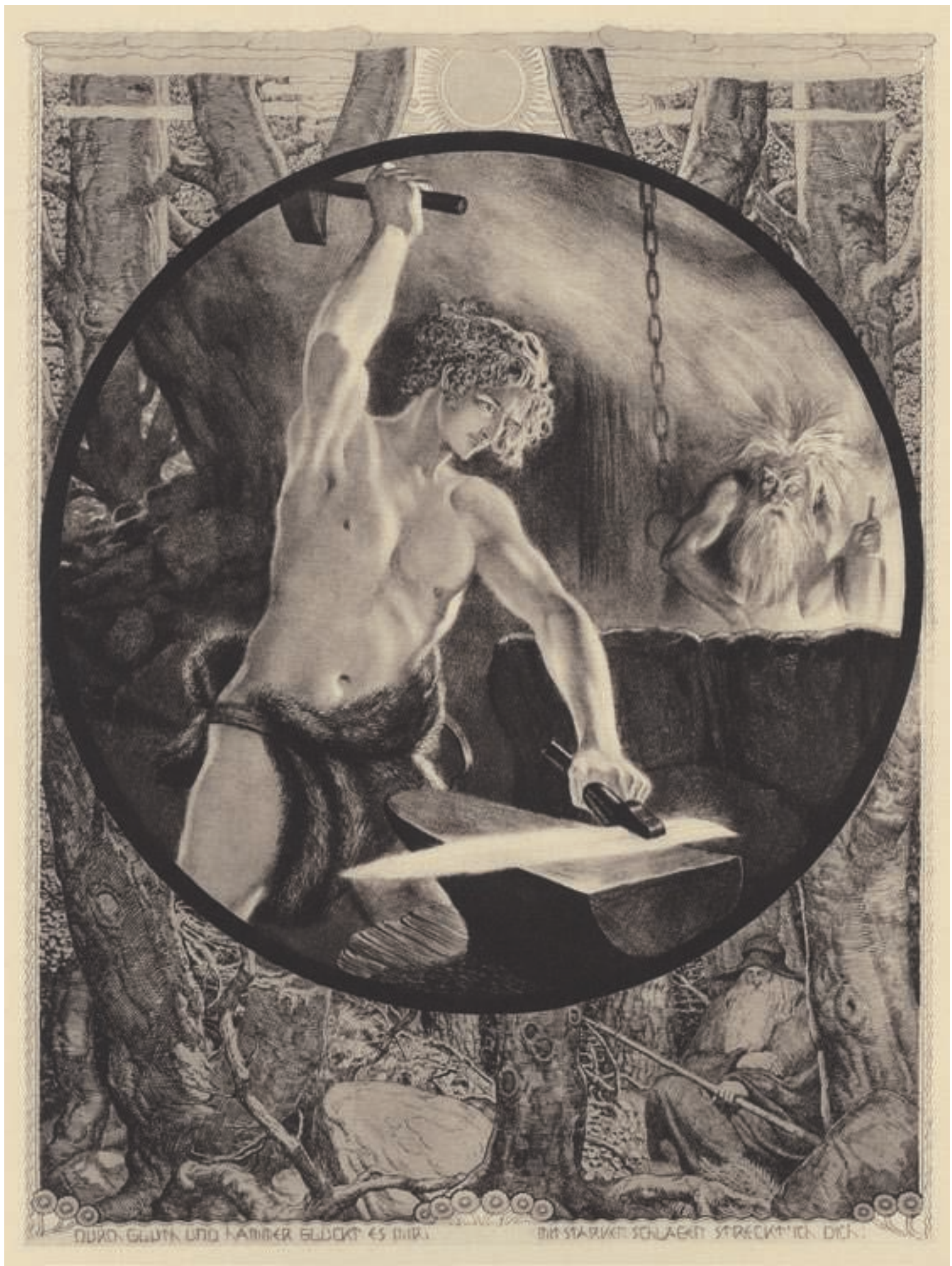

Soixante et unième planche du cycle Der Ring des Nibelungen :Jung-Siegfried (Berlin, Ludwig Schroeter, 1930 ; coll. BNU) 
baignade de Siegfried nu à la fin du premier chant du film rappelle très nettement la gravure correspondante de Stassen ; de même, d'une œuvre à l'autre, les décors de l'antre du dragon se ressemblent étonnamment, et l'on pourrait multiplier les exemples.

Le Ring de Stassen a également retenu l'attention d'Udo Bermbach, l'un des plus éminents spécialistes allemands de Wagner et du wagnérisme. Dans son dernier ouvrage ${ }^{11}$, il se penche sur la figure de Siegfried créée par Stassen et sur son arrière-plan idéologique. Il montre qu'elle est essentiellement conforme à l’image héroïque véhiculée par le cercle des Bayreuther Blätter. Stassen représente un héros innocent et pur, en harmonie avec la nature. La nudité partielle ou entière du personnage fait de lui un être sain, à la jeunesse et au corps épanouis, mais fragile face à Hagen qui affiche son harnachement guerrier et sa nature d'assassin. Au moment de mourir, Siegfried ploie sous le coup tel un arc tendu, lève les bras au ciel, nimbé de rayons solaires. Cette imagerie, certes naïve, mais malgré tout moins grandiloquente que chez bien d'autres illustrateurs wagnériens, n'est peut-être pas celle que l'on s'attendrait à trouver en ce début de $20^{\mathrm{e}}$ siècle, surtout à partir de 1914. Elle ne correspond évidemment pas - est-ce nécessaire de le préciser ? - au Siegfried de Wagner. Stassen ne fait presque rien transparaître des errements du personnage. Son Siegfried n'a pas besoin d'être rédimé par l'ultime souvenir amoureux de Brünnhilde puisqu'il conserve sa pureté tout au long de son parcours. Il reste à l'écart de la corruption du monde au lieu, comme chez Wagner, d'en être l'un des rouages, fût-ce malgré lui. Mais le héros de Stassen, très proche de l'esprit Jugend et de la "Lebensreform " très en vogue dans toute l'Allemagne à la fin du $19^{e}$ siècle, ne répond pas non plus, comme le démontre Bermbach, à l'image belliqueuse du défenseur de la germanité, à l'incarnation de la force d'un peuple, une représentation de Siegfried que l'on ne manquera pas d'exploiter dès la Première Guerre mondiale.

Le cycle de Stassen vise avant tout à célébrer une nature originelle et la nudité, féminine comme masculine. Le résultat est souvent esthétiquement spectaculaire: les planches les plus originales sont celles qui figurent le frêne du monde, les eaux du Rhin et celles, utopiques et immémorielles, qui baignent les rêves d'Erda. La nudité semble harmoniser dieux et hommes dans une même plastique. Des Nornes qui s'enlacent audessus du frêne à Woglinde, personnage central d'une gravure, à Freia " die holde " sortie tout droit d'une couverture de Jugend, sans oublier bien sûr Sieglinde et Brünnhilde, toutes participent par leur nudité ou seminudité à la célébration du corps. Du côté du masculin, les dieux Loge, Donner et Froh ne sont pas en reste et leurs corps dessinent déjà celui du futur Siegfried. Le cas de Loge est particulièrement remarquable. Stassen gomme tout ce qu'il peut y avoir de double ou d'inquiétant en lui ; il en façonne l'image à partir du récit que fait le personnage de sa rencontre avec les filles du Rhin dans la deuxième scène du Rheingold. Loge y dresse le tableau très lucrétien de la nature tout entière animée par le désir, où règnent partout le charme et l'attrait de la femme. Un bon prétexte est offert à Stassen pour faire triompher les corps. Dans la gravure 11 du cycle consacré à cet opéra, tous les personnages déploient leur nudité : Loge lui-même aux côtés des filles du Rhin, ainsi qu'une multitude de couples insérés dans l'encadrement de la scène. Mais il y a nudité et nudité. Celle-là, comme on l'a dit pour la représentation de Siegfried, n'a rien de l'aspect martial omniprésent dans l'imagerie nazie et dans les œuvres plus tardives de Stassen.

Un rêve d'innocence traverse donc le Ring de Stassen. Dieux et héros en sont les pures émanations. On ne voit pas Wotan aveuglé par le désir de puissance et de richesse enfreindre ses propres lois, trahir sa parole. Chez Stassen, seuls Alberich et Hagen agissent pour la perte du monde. On en oublierait que Wotan, chez Wagner, mutile et fait abattre l'arbre du monde et que c'est lui le premier à ne pas vouloir rendre l'or aux filles du Rhin. De même Stassen édulcore souvent la violence. C'est à peine si l'on remarque que Freia est poursuivie par les Géants, et la mort de Siegmund paraît bien statique comparée à celle gravée par Hugo Braune (très semblable pourtant dans sa scénographie), pour ne mentionner que ces exemples... Cette idéalisation systématique amène l'artiste à traiter plus allusivement le contenu du Götterdämmerung, voire à l'éluder. Pour les mêmes raisons, il marginalise la représentation du monde social (pas une seule gravure ne fait apparaître le séjour des Gibichungen).

Si par de tels choix les gravures de Stassen se tiennent à l'abri d'une idéologie belliciste, elles ne manquent pas par ailleurs de dissoudre le contenu révolutionnaire et politique de l'œuvre de Wagner.

On aboutirait sans doute à une conclusion semblable si l'on se penchait sur l'idéologie christique qui sous-tend le cycle de Parsifal. Dans la mouvance de 
Wolzogen et de son cercle, Stassen propose une vision religieusement univoque : le héros Parsifal, au départ jeune homme à la moustache wilhelminienne, prend les traits d'Amfortas, de Titurel puis du Christ lui-même tandis qu'une symbolique uniquement chrétienne envahit le champ visuel, non sans une grande habileté esthétique, comme on l'a vu. Stassen ne cherche pas à faire seulement de Parsifal un personnage empathique qui traverse des générations de souffrance; il l'assimile au Christ, le circonscrit dans l'espace symbolique du dogme chrétien. L'idéal wagnérien est pris à contre-pied. Alors que Wagner voyait en l'art un moyen de retrouver la force universelle d'un Christ que les religions avaient dévoyé, Stassen anime une image pieuse, bien dans l'esprit des Bayreuther Blätter. Le Christ nazaréen de Stassen se substitue à la figure chrétienne mâtinée de théories sociales et de sagesse bouddhique que Wagner était allé chercher de façon parfois très anticléricale chez Feuerbach et plus tard chez Schopenhauer ${ }^{13}$. Ainsi Stassen reconduit le mythe dans le champ du religieux au lieu d'emprunter le chemin inverse tracé dans l'œuvre musicale de Wagner et théorisé dans ses écrits zurichois comme plus tard dans son essai sur l'art et la religion ${ }^{14}$.

C'est aussi sous cet angle que l'on peut examiner les suites gravées de Stassen. Elles témoignent de l'écart idéologique que le cénacle de Bayreuth manifeste dès l'origine avec l'œuvre de Wagner, alors même qu'il s'en proclame le fidèle héritier. Cet écart n'empêche en aucune façon d'apprécier l'illustrateur qu'est Stassen dans - et peut-être avant tout dans - les cycles que nous avons évoqués : grâce à un chassé-croisé d'esthétiques, Stassen y trouve une identité d'artiste.

\section{Jean-Louis Elloy}

\section{ORIENTATIONS BIBLIOGRAPHIQUES :}

STASSEN FRANZ | Parsifal. 15 Bilder zu Richard Wagner's Bühnenweihfestspiel. - Berlin, B. Behr's Verlag, 1914

STASSEN FRANZ / Tristan und Isolde. 12 Bilder zu Richard Wagners Tondichtung. - Berlin, Fischer und Franke, s. d. [1899]

STASSEN FRANZ | Der Ring des Nibelungen: Rheingold. - Berlin, Verlag Ludwig Schroeter, s. d. [ca. 1930]

STASSEN FRANZ / Der Ring des Nibelungen : Jung-Siegfried. - Berlin, Verlag Ludwig Schroeter, 1930

STASSEN FRANZ | Mein Leben. - Mainz, 1984

BERMBACH UDO / Richard Wagner in Deutschland. Rezeption. Verfälschungen. - Stuttgart, 2011

BERMBACH UDO / Der Wahn des Gesamtkunstwerks : Richard Wagners politisch-ästhetische Utopie. - Stuttgart, 2004

MERK ANTON / Franz Stassen : 1869-1949 ; Maler, Zeichner, Illustrator ; Leben und Werk. - Hanau, 1999

MEYER STEPHEN C. | Illustrating Transcendence : Parsifal, Franz Stassen, and the Leitmotif, in The Musical quarterly, août 2009 


\section{Notes}

1 - Wagner, le "Ring " en images : autour de la collection Bruno Lussato. Paris, Bibliothèque nationale de France, 1994

2 - L'exposition réalisée par le Musée d'art et d'histoire de Genève en 2005 , Richard Wagner : visions d'artistes, n'a pas montré d'œuvres de Stassen. Son catalogue cependant le mentionne brièvement et le définit comme "l'enlumineur de la geste wagnérienne dans les années 1900-1930 ", dont on peut admirer la mise en page et les arabesques art nouveau, mais dont la production très inégale sombre vite dans " la vulgarité " dès qu'il passe à la toile.

3- En 1935, la chancellerie du Reich passe commande auprès de Stassen d'un ensemble de quatre tapisseries illustrant la légende d'Edda. Elles sont réalisées d'après ses aquarelles mais exposées peu de temps car elles ne sont pas appréciées des dignitaires du régime. Cet épisode est révélateur : il montre à quel point il s'avère difficile pour Stassen de s'adapter à l'esthétique agressive alors en vigueur. A partir des années 1940, il prend ses distances avec la propagande nazie. Il est possible que l'engagement dans la résistance au nazisme de sa fille et de son beau-fils, desquels il est toujours resté proche, ait joué un rôle dans cette tardive discrétion.

4- Hans von Wolzogen, écrivain, est aux côtés de Houston Stewart Chamberlain l'une des figures les plus importantes du premier cercle wagnérien. A partir de 1878, il dirige les Bayreuther Blätter, organe de presse censé promouvoir l'œuvre et les idées de Wagner.

5- Cf. la lettre de Stassen datée du 7 octobre 1938 et adressée à Boulet (lettre que possède le fonds acquis par la BNU) : " Es ist meine Lebensaufgabe gewesen Richard Wagners Werke in der Graphik auszudrücken " (illustrer l'œuvre de Wagner a été la tâche essentielle de ma vie).

6- La BNU possède l'édition originale, parue en 1899, de Tristan und Isolde, une édition de 1914 de Parsifal, avec un poème de Wolzogen en épigraphe, et une édition du Ring en trois volumes (Das Rheingold, Die Walküre, JungSiegfried), édition antérieure à 1938, dédicacée par Stassen qui a également signé chacune des planches.

7 - Dans son article Illustrating Transcendence : Parsifal, Franz Stassen, and the Leitmotif (le seul, à notre connaissance, consacré à ce cycle de Stassen), Stephen C. Meyer montre, en s'appuyant sur de nombreux exemples, que les leitmotive musicaux repris par l'artiste dans ses planches sont directement empruntés aux guides thématiques de Wolzogen, publiés dans les années 188o. Meyer analyse comment Stassen intègre les citations de la partition à l'image, et retravaille graphiquement la plastique de la notation musicale pour lui donner un tour médiéval (à l'instar des éditions de chant grégorien publiées à la fin du $19^{\mathrm{e}}$ siècle). Stassen cherche ainsi à faire de son portfolio un véritable livre d'heures " fin-de-siècle ». Le leitmotiv y acquiert une vertu mystique et s'inscrit dans une conception religieuse d'ensemble. Sa fonction, que Meyer qualifie de métonymique, va donc bien au-delà du rôle illustratif que lui attribue Wolzogen.

8 - « Selig in Liebe für den Tempelbauer/Zeigt er der Zeit im Raumgebild den Gral » (qu'il soit bienheureux l'architecte du temple; il a donné espace au temps et y a montré le Graal). L'expression " Tempelbauer » (l'architecte du temple) peut ici tout aussi bien désigner Wagner bâtisseur de Bayreuth que Stassen qui en reproduit graphiquement la construction.

9 - D'après Stephen C. Meyer (cf. son article cité plus haut), de tous les artistes de la fin du $19^{\mathrm{e}}$ siècle, c'est Alphonse Mucha que l'on peut considérer comme le plus proche de Stassen, tout au moins dans son Parsifal. Il préfère d'ailleurs l'expression française d' " art nouveau » à celle de "Jugendstil » pour définir l'esthétique de ce cycle wagnérien. L'influence de Mucha nous paraît cependant beaucoup plus indirecte que celle des artistes allemands que nous avons mentionnés.

10-Anton Merk, Franz Stassen (1869-1949). Maler, Zeichner, Illustrator. Leben und Werk. - Hanau, 1999, p. 20-2
11- Udo Bermbach, Richard Wagner in Deutschland. Rezeption. Verfälschungen. - Stuttgart : Metzler, 2011, p. 351 à 359

12-Dans les rééditions en plus petit format du Ring de Stassen (dès 1930), le cahier Götterdämmerung disparaît et le cycle composé de trois volets s'achève avec le cahier Jung-Siegfried.

13- Pour une comparaison entre Feuerbach, Marx et Wagner sur la question du religieux, cf. Udo Bermbach, Der Wahn des Gesamtkunstwerks (Metzler, 2004) p. 94 à 103 et p. 314 à 336. Sur l'influence de Schopenhauer et du bouddhisme sur le christianisme de Wagner et l'abondante bibliographie consacrée à ce sujet, cf. ibid., p. 286 et suivantes.

14-Richard Wagner, Die Kunst und die Revolution, Das Kunstwerk der Zukunft, Oper und Drama (1849/1851), Religion und Kunst (1880) 